\title{
APLICATIVOS PARA O ENSINO DE ESTATÍSTICA: AVALIAÇÃO E REFLEXÕES SOBRE SEU USO
}

\author{
Paulo Marcos S. Ribeiro \\ Universidade Federal de Pernambuco, Brazil \\ pmribeirogen2@hotmail.com
}

Com a ênfase que se tem hoje em inserir tecnologia digital na educação, para auxiliar no trabalho do professor e na aprendizagem do estudante, o uso de tablets, smartphones, software, aos poucos estãosendo inseridos no espaço escolar, além de auxiliar nas diversas atividades da nossa vida social. Portanto, o estudo tem como objetivo criar um instrumento para avaliar aplicativos voltados para o ensino de Estatística na Educação Básica, avaliando as dimensões técnicas, pedagógicas e estatística desses software. Assim, buscamos identificar aplicativos com sistema Android, na condição que os mesmos fossem gratuitos e no idioma português, em julho de 2014, identificando assim, apenas três aplicativos. Na análise, verificamos limitações nos aplicativos nas três dimensões. Contudo, o instrumento ainda aponta o que o professor pode verificar para contemplar na sua aula o que o recurso tem a contribuir na prática no ensino de estatística.

\section{INTRODUÇÃO}

Com o avanço e o acesso das tecnologias digitais móveis, esse termo é citado em Borba, Silva \& Gadanidis (2014) para as tecnologias como internet, celulares e tablets. Iremos utilizá-lo para os dispositivos móveis, assim como para os softwares inseridos nos mesmos, como tablet, smartphones, notebooks e software, têm oportunizando a construção de novos meios de acesso para o uso da informação e possibilitando a produção de conhecimentos, como uma construção dinâmica por meio de textos como mensagens, imagens e sons, em um único dispositivo. No contexto da educacional, essa realidade têm atualmente mudado aos pouco, com a inserção de algumas dessas tecnologias digitais, com tablet e computadores, inseridos no ambiente escolar no processo de ensino e aprendizagem.

Para a Organização das Nações Unidas para a Educação, a Ciências e a Cultura - UNESCO (2013) as tecnologias digitais móveis podem ampliar e enriquecer oportunidades educacionais aos estudantes nos diversos ambientes. Com os aparelhos móveis - em particular os smartphones e tablets - possibilitam estar presente em todos os lugares e podem ser utilizados por alunos e educadores em todo o mundo. Além disso, o seu uso tem a chance de realizar práticas educativas que possam inovar o ensino e a aprendizagem na sala de aula ou fora dela.

Estudos como de Franklin \& Peng (2008); Batista (2011); Pereira, Schuhmarcher, Schuhmarcher, \& Dalfovo, (2012), verificou-se no ensino e aprendizagem de Matemática, que as tecnologias com o uso de aplicativos específicos podem potencializá-lo, favorecendo na visualização, na manipulação de dados e colaboração entre alunos dentro e fora da sala de aula.

Assim, como motivação para a elaboração desse estudo versou no fato da ênfase que se tem hoje em inserir a tecnologia digital na educação, dos governos estaduais, no Brasil, que vem desde 2012, distribuindo, tablets aos professores do ensino médio da rede pública de ensino, como já têm sendo inseridas algumas redes públicas municipais de ensino, para auxiliar no trabalho do professor e na aprendizagem do estudante. Com isso, o estudo tem como objetivo criar um instrumento e avaliar aplicativos disponíveis com potencial para o ensino de Estatística na Educação Básica em língua portuguesa.

\section{CONTEXTO E PERCURSO DO INSTRUMENTO DE AVALIAÇÃO}

Para elaboração de um instrumentoque visamosavaliar os aplicativos, buscamos concretizálo a partir daliteratura na área, numa revisão sobre avaliação de software com critérios que foram organizados mediante propostas de autores como: Oliveira, Costa, \& Moreira (2001); Bednarik (2002); Atayde, Teixeira, \& Pádua (2003); Prates \& Barbosa (2003); Borba (2010) e Silva (2012). E para parte específica de Estatística, buscamos em documentos oficiais de ensino como os Parâmetros Curriculares de Matemática de Pernambuco (2012). Assim, o instrumento de avaliação, foi construído com uma parte inicial que solicitainformações para identificação do aplicativo e as três partes de avaliação: dimensão técnica; dimensão didático-pedagógica e dimensão estatística.

In: M.A. Sorto (Ed.), Advances in statistics education: developments, experiences and assessments. Proceedings of the Satellite conference of the International Association for Statistical Education (IASE), July 2015, Rio de Janeiro, Brazil. 
Com isso,elencamos as informações para saber quais os requisitos relevantes que contemplassem os aplicativos.

No instrumento,na Dimensão Técnica busca-seinformações que o usuário deve ter sobre o aplicativo de modo que contribuam e auxiliem o usuário no conhecimento e aplicação do programa.

$\mathrm{Na}$ Dimensão Didático-Pedagógica, pretendemos nos critérios identificaros objetivos didáticos ou maneira de condução no processo ensino aprendizagem com uso dos aplicativos, no apoio ao professor como do aluno.

Como métrica para avaliar para essas duas categorias, dispomos como: $0=$ (não contempla); $1=$ (contempla parcialmente); $2=($ contempla $)$, nos critérios analisados para as categorias dessasdimensões.

Com a Dimensão Estatística, identificamosno programa Parâmetros Curriculares de matemática de Pernambuco (2012) como base do componente curricular para o ensino na Educação Básica de matemática,e na literatura estudos sobre o contexto envolvendo a aprendizagem dos conceitos envolvendo o ensino da Estatística. Como métrica para avaliar, dispomos de $0=$ (não contempla) e $1=$ (contempla), aos conteúdos inseridos nos aplicativos para essas categorias.

Construído o modelo de avaliação,organizado em quatro quadros, realizamos uma buscapara identificar os aplicativos Android no Google Play Store, em julho de 2014, tendo como palavra indutora: estatística, com a condição que o aplicativo fosse gratuito, em idioma português.Desse modo foram identificamos 9 (nove) aplicativos que fossem viáveis de acordo para o ensino de Estatística, portanto, dentre esses, apenas 3 (três) com os critérios estabelecidos para o nosso estudo, gratuito e no idioma português. Sendo os seguintes aplicativos: 1-Calculadora Estatística (Free); 2- Calculadora Estatística ++ e 3- Estatística Fácil.Os demais apresentavam no idioma da língua inglesa, em que neste estudo não serão contemplados para análise.

Assim, apresentaremosos quadroscom resultados geral da análise desses aplicativos, apontando suas aplicabilidades como suas limitações para uso em sala de aula, possibilitando reflexões sobre esses recursos que sendo inseridos aos poucos no contexto educacional.

\section{INSTRUMENTO E AVALIAÇÃO DOS APLICATIVOS DE ESTATÍSTICA}

Com o instrumento identificamos os critérios de cadacategoria atribuindoaos aplicativosrespectivas métricas a serem utilizadas na avaliaçãonas dimensões técnica, didáticopedagógica e de Estatística.Iniciamos com a avaliação dos aplicativos pela sua parte na Dimensão Técnica.

De acordo comaanálise dos aplicativos na Documentação, todos os aplicativos não apresentaram aspectos relevantes sobre informações a respeito do uso do aplicativo, conteúdo, o mesmo ocorrendo como os aspectos educacionais, que não apresenta nenhuma informação educacional.

$\mathrm{Na}$ Navegabilidade, o aplicativo Calculadora Estatística (Free), possibilita de maneria contempla satisfatoriamente esse categoria, no uso de manipulação, com fácil acesso de utilizar partes do aplicativo. Os demais, não contemplam de maneira satisfatória, faltando alguns dos critérios nos itens analisados, a possibilidade retorna e rever o que foi realizado pelo usuário. $\mathrm{Na}$ verificação da Interface, os aplicativos apresentam imagens com qualidade, portanto não apresentam animações, como também links e recursos sonoros. E para finalizar essa dimensão, verificamos nas versões utilizadas nãopermitem compartilhar ou gravar sua produção.

Percebe-se as limitações dos aplicativos em vários aspectos analisados, tendo em vista os critérios utilizados na avaliação. Como veremos no Quadro 1 , o quadro de avaliação da parte técnica, a seguir. 
Quadro 1: Quadro de avaliação da Dimensão Técnica.

\begin{tabular}{|c|c|c|c|}
\hline $\begin{array}{l}\text { Documentação - Apresenta informações relevantes e objetivas } \\
\text { para uso do ponto de vista: }\end{array}$ & 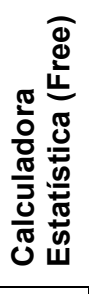 & 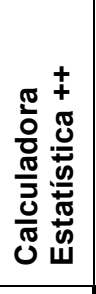 & 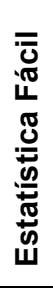 \\
\hline Técnico & 1 & 1 & 1 \\
\hline Educacional & 0 & 0 & 0 \\
\hline Estatístico & 1 & 1 & 1 \\
\hline \multicolumn{4}{|l|}{ Navegabilidade } \\
\hline $\begin{array}{l}\text { Oferece ao estudante opções para interromper, retornar e iniciar } \\
\text { atividades ao manipular a ferramenta? }\end{array}$ & 2 & 0 & 1 \\
\hline $\begin{array}{l}\text { Permitem acessar de maneira fácil todas as partes do aplicativo? } \\
(0=\text { não; } 1=\operatorname{sim})\end{array}$ & 1 & 1 & 0 \\
\hline \multicolumn{4}{|l|}{ Interface } \\
\hline $\begin{array}{l}\text { Possui imagens com qualidade e animações que contribuem para } \\
\text { compreensão do conteúdo trabalhado? }\end{array}$ & 1 & 0 & 1 \\
\hline Utiliza recursos sonoros? & 0 & 0 & 0 \\
\hline $\begin{array}{l}\text { Possui ícones, botões, abas e links que podem contribuir para } \\
\text { facilitar na ação do usuário? }\end{array}$ & 1 & 1 & 1 \\
\hline \multicolumn{4}{|l|}{ Compartilhamento e Memória da produção } \\
\hline $\begin{array}{l}\text { Permite importar e exportar dados de algum outro software, } \\
\text { como planilha eletrônica ou processador de texto? }\end{array}$ & 0 & 0 & 0 \\
\hline $\begin{array}{l}\text { Permite gravar e carregar os dados inseridos para continuar o } \\
\text { processo posteriormente? }\end{array}$ & 0 & 0 & 0 \\
\hline
\end{tabular}

$\mathrm{Na}$ parte didático-pedagógica, apenas três categorias foram contempladas na avaliação como vemos na análise dessa dimensão a seguir.

$\mathrm{Na}$ categoria Interação, todos permitem inserir os dados. Tendo em vista que nos demais critérios os aplicativos não oferece algum tipo de feedback, para o usuário, somente a resposta como resultado dos cálculos. Em relação à abordagem da linguagem, identificarmos ser adequado, apenas os conceitos abordados sabendo que os mesmos, não apresentam texto, e sim uma lista dos conteúdos. Logo, os três aplicativos apresentam conteúdo para o ensino médio, assim como dentre esses ainda verificamos um para os anos iniciais e dois para anos finais.

$\mathrm{Na}$ categoria Acessibilidade não foi contemplada por esses aplicativos. $\mathrm{Na}$ Abordagem/Diferencial, identificamos que um dos aplicativos representa seus dados em gráficos: Calculadora Estatística (Free). O aplicativo Estatística Fácil, representa seus dados em tabela, sendo o que diferencia no processo utilizado com o lápis e papel desses aplicativos.

Nas demais categorias, verificamos que existe a necessidade aplicação de uma abordagem pedagógica para que os mesmo com suas limitações que possam atua de maneira mais pedagógica ao serem utilizados na sala de aula, no contexto de apoio tanto ao professor como o aluno, ao invés de serem utilizados intuitivamente. A seguir veremos os resultados no Quadro 2, o quadro da Dimensão Didático-Pedagógica. 
Quadro 2: Quadro de avaliação da Dimensão Didático-Pedagógica.

\begin{tabular}{|c|c|c|c|}
\hline DIMENSÃO DIDÁTICO-PEDAGÓGICA & 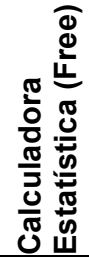 & 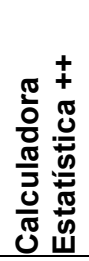 & 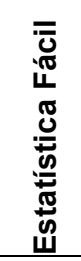 \\
\hline \multicolumn{4}{|l|}{ Interação } \\
\hline Permite que o usuário insira dados? & 2 & 2 & 2 \\
\hline $\begin{array}{l}\text { Fornece feedback imediato na entrada de dados do } \\
\text { estudante? }\end{array}$ & 0 & 0 & 0 \\
\hline Possibilita a interação no trabalho em grupo? & 0 & 0 & 0 \\
\hline \multicolumn{4}{|l|}{ Linguagem } \\
\hline $\begin{array}{l}\text { Utiliza uma linguagem adequada para o desenvolvimento } \\
\text { dos conceitos estatísticos? }\end{array}$ & 0 & 0 & 0 \\
\hline É adequado a partir de que nível de escolaridade: (AI) Anos & AF/ & $\mathrm{Al} /$ & $\mathrm{AF} /$ \\
\hline Iniciais; (AF) Anos Finais e (EM) Ensino Médio? & EM & EM & EM \\
\hline \multicolumn{4}{|l|}{ Acessibilidade } \\
\hline $\begin{array}{l}\text { Inclui elementos para permitir acesso a pessoas portadoras } \\
\text { de alguma deficiência }\end{array}$ & 0 & 0 & 0 \\
\hline \multicolumn{4}{|l|}{ Abordagem /Diferencial } \\
\hline $\begin{array}{l}\text { O aplicativo traz algo que promove um diferencial àquilo que } \\
\text { possa ser feito sem essa tecnologia? }\end{array}$ & 2 & 1 & 2 \\
\hline \multicolumn{4}{|l|}{ Fundamentos Pedagógicos } \\
\hline $\begin{array}{l}\text { Possui guia de apoio pedagógico para o professor, } \\
\text { apresentando informações sobre objetivos didáticos e } \\
\text { conteúdos a serem trabalhados como apoio ao professor? }\end{array}$ & 0 & 0 & 0 \\
\hline Explicita a concepção de aprendizagem? & 0 & 0 & 0 \\
\hline
\end{tabular}

$\mathrm{Na}$ dimensão Estatística, a análise nos demonstra os resultados da avaliação, no qual verificamos que dentre os três aplicativos, um apresenta os dados em gráficos e outro representa seus dados em tabela. Nos elementos constitutivos de gráficos e tabelas, modificar à escala, associar tabela a gráfico, não foram contemplados nesses aplicativos.Apenas o aplicativo Calculadora estatística (Free), apresenta amostra e população e comparar dados. O aplicativo Estatística Fácil apresenta frequência absoluta/relativa e contemplam frequência acumulada. Portanto, todos contemplam conteúdos de medidas de tendência central, tendo ao menos uma das medidas, como média aritmética, moda e a mediana. O mesmo fato ocorrendo com as medidas de dispersão. Supomos que essas medidas mais usuais e comum de ser tratada no ambiente escolar,foram mais abordadas pelos desenvolvedores, por isso foram abordadas em todos os aplicativos.

Nos aplicativos foi verificado que apenas trabalham com a única variável, a numérica, não sendo uma prioridade de abordá-la outro tipo de variável. O mesmo ocorrendo com a possibilidade de classificação para os dados. Como veremos no Quadro 3, a seguir. 
Quadro 3: Quadro de avaliação da Dimensão Estatística.

\begin{tabular}{|c|c|c|c|}
\hline DIMENSÃO ESTATÍSTICA & 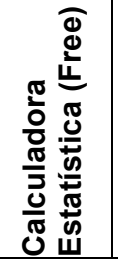 & 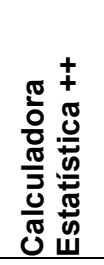 & 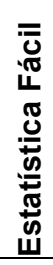 \\
\hline \multicolumn{4}{|l|}{ Conteúdos } \\
\hline Representação em gráfico & 1 & 0 & 0 \\
\hline $\begin{array}{l}\text { Tipo de gráfico: (0) Não apresenta; (1) Barra; (2) Linha; (3) } \\
\text { Setor; (4) Histograma; (5) Dispersão; (6) BoxPlot }\end{array}$ & $\begin{array}{c}1 ; 2 ; 4 \\
6 \\
\end{array}$ & 0 & 0 \\
\hline Permite modificação da escala & 0 & 0 & 0 \\
\hline Representação como tabela & 0 & 0 & 1 \\
\hline Associa tabelas a gráficos. & 0 & 0 & 0 \\
\hline $\begin{array}{l}\text { Possibilita acrescentar ou alterar elementos constitutivos de } \\
\text { gráficos e tabelas (título, fonte, escalas etc) }\end{array}$ & 0 & 0 & 0 \\
\hline Amostra e População & 1 & 0 & 0 \\
\hline Frequência simples & 1 & 0 & 1 \\
\hline Frequência Absoluta/Relativa & 0 & 0 & 1 \\
\hline Frequências Acumuladas & 0 & 0 & 1 \\
\hline Compara os conjuntos de dados & 1 & 0 & 0 \\
\hline Medidas de Tendência Central & 1 & 1 & 1 \\
\hline Medida de Dispersão & 1 & 1 & 1 \\
\hline Tipos de variáveis: (1) numérica e (2) categórica & 1 & 1 & 1 \\
\hline Possibilita classificação de dados a partir de critérios do usuário & 0 & 0 & 0 \\
\hline
\end{tabular}

Os resultados das análises verificaram que os aplicativos não apresentam informações que contribuíssem com o usuário para explorá-lo de maneira contextualizada, para o ensino da Estatística, apresentando somente resultados dos cálculos, das medidas estatísticas, prontos, o que não promove uma autonomia do aluno para resolução de atividades.

\section{CONSIDERAÇÕES FINAIS}

A inserção de dispositivos móveis como tablet nas escolas, possibilita assim como os livros de avaliações, contribuindo para o professor reconhecer aspectos importantes na parte técnica, didático-pedagógica e a específica, dessas ferramentas comorecurso pedagógico.

Acreditamos que no instrumento criado e na avaliação desses aplicativos, possibilitamos ampliar a implantação de aplicativos como de outros software na sala de aula e como recurso de avaliação para o professor, apontando suas limitações e eficiência nos critérios criados. Assim, os dispositivos, passam a ser recursosmenos vazios de conteúdospara sua utilização na prática educativa.

O modelo criado como instrumento de avaliação, possibilita que sejam feitasreflexões nesses tipos de recursos digitais, como numa abordagem didático-pedagógica, que visano processo de interação do estudante, no contexto com a aprendizagem, como sujeito de seu conhecimento. Assim como oa importância de observar a parte curricular que se propõe trabalhar, oportunizandoa atender na sua interface a melhor maneira de proporcionar ao usuário uma boa qualidade tecnológica que possa explorar os conteúdos abordados.

Percebemos que existe ainda a necessidade de novos aplicativos em língua portuguesa, tendo em vista o número reduzido de aplicativos encontrados no Play Store, tendo no Brasil uma 
gama de usuários que possam utilizar esses recursos, a ser utilizado tanto nostablets como nos smartphones.

Portanto, a avaliação nos mostra as limitações nas três dimensões que identificamos nos aplicativos analisados, em que fica clara a necessidade da atualização e criação de outros aplicativos que possam auxiliar aos alunos a aprenderem conceitos estatísticos. Além disso, o instrumento ainda aponta o que o professor pode verificar para contemplar na sua aula com o recurso e oque tem a contribuir na prática no ensino de estatística.

\section{REFERÊNCIAS}

Atayde, A. P. R., Teixeira, A. B. M., \& Padua, C. I. P. S. (2003). MAQSEI - uma Metodologia de Avaliação de Qualidade de Software Educacional. In XIV SBIE Simpósio Brasileiro de Informática na Educação, NCE/UFRJ, Rio de Janeiro.

Batista, S. C. F. (2011). M-learnMat: modelo pedagógico para atividades de m-learning em Matemática. Tese (Doutorado) - Universidade Federal do Rio Grande do Sul. Centro de Estudos Interdisciplinares em Novas Tecnologias na Educação. Programa de Pós-Graduação em Informática na Educação. Porto Alegre, RS. 225f.

Bednarik, R. (2002). Evaluation of Education Environments: The TUP Model. MSc. Thesis, Department of Computer Science, University of Joensuu, Finland.

Borba, M. C. (2010). Softwares e internet na Sala de Aula. In X ENEM - Encontro Nacional de Educação Matemática, Salvador. X ENEM.

Borba, M. C., Scucuglia, R., \& Gadanidis, G. (2014). Fases das Tecnologias Digitais em Educação Matemática: Sala de aula e internet em movimento. 1. ed. Belo Horizonte: Autêntica. 149p.

Franklin,T., \& Peng, L. W. (2008). Mobile math: math educators and students engage in mobile learning. J Comput High Educ., 20, 69-80.

Oliveira, C. C., Costa, J. W., \& Moreira, M. (2001). Ambientes informatizados de aprendizagem: produção e avaliação de software educativo. Campinas: Papirus.

Pernambuco (2012). Secretaria de Educação. Parâmetros para a educação básica do Estado de Pernambuco: parâmetros curriculares de Matemática para o Ensino Fundamental e Médio. Pernambuco: CAEd/Ufjf

Pereira, L. R., Schuhmacher, V. R. N., Schuhmacher, E., \& Dalfovo, O. (2012). O uso da tecnologia na educação, priorizando a tecnologia móvel. In IV Seminário Nacional de Educação Profissional e Tecnológica. CEFET. MG.

Prates, R. O, \& Barbosa, S. D. J. (2003) . Avaliação de Interfaces de Usuário - Conceitos e Métodos. In Juan Manuel Adán Coello; Sandra C. P. Ferraz Fabbri. (Org.). Jornada de Atualização em Informática do Congresso da Sociedade Brasileira de Computação. Campinas: SBC, 2003, v. 2, p. 245-293.

Silva, A. C. B. (2012). Softwares educativos: critérios de avaliação a partir dos discursos da interface, da esfera comunicativa e do objeto de ensino. 1. ed. Recife: Editora Universitária, v. $1.332 \mathrm{p}$.

UNESCO (2013). Policy Guidelines for Mobile Learning.Paris, France, 41p. 